have received a red cell transfusion. Data is entered onto an online proforma and reported back in a format that allows each hospice to compare its own performance against national results. The reports act as a benchmark, allowing hospices to evaluate their performance against pre-determined standards.

Results Results from other, similar national comparative audits have shown that there is room for improvement in compliance with evidence based patient blood management practise, particularly in respect of testing for anaemia and in the area of patient safety. We expect this audit to produce similar results.

Conclusion The audit will demonstrate areas of practice which are amenable to change, particularly in the spheres of anaemia investigation and management, as well as patient safety. These include being sure of the reason for transfusion and the benefits the patient will derive, making the patients aware of the risks and alternatives to transfusion and ensuring that patients are adequately monitored especially with the increasing awareness of the prevalence of Transfusion Associated Circulatory Overload.

\section{P-54 WAS IT WORTH IT? INTRATHECAL ANALGESIA FOR CANCER PAIN; THE VIEWS OF CARERS}

\footnotetext{
1,2 Melanie Huddart, ${ }^{3}$ Nishi Patel, ${ }^{4}$ Helen Makins, ${ }^{5}$ Theresa Mitchell, 1,2 Jane L Gibbins, ${ }^{2} J u a n$ Graterol, ${ }^{1,2}$ Deborah Stevens, ${ }^{4,6}$ Paul Perkins. ${ }^{1}$ Cornwall Hospice Care, Hayle, UK; ${ }^{2}$ Royal Cornwall Hospitals NHS Trust, UK; ${ }^{3}$ University Hospitals Bristol, UK; ${ }^{4}$ Gloucestershire Hospitals NHS Foundation Trust, UK; ${ }^{5}$ University of Worcester, UK; ${ }^{6}$ Sue Ryder Leckhampton Court Hospice, UK
}

\subsection{6/bmjspcare-2017-00133.54}

Background Intrathecal Drug Delivery (ITDD) is known to reduce pain in patients where conventional systemic analgesia has been ineffective or intolerable. However more information about how this intervention affects quality of life (QOL) and function in those with advanced incurable cancer is required. Aim To explore the views of bereaved carers regarding the physical and psychosocial effects of ITDD in patients with advanced incurable cancer.

Methods Qualitative interviews were undertaken with carers of deceased individuals who received ITDD (percutaneous, external system) as part of their pain management. Interviews were analysed using thematic analysis.

Results Eleven interviews were conducted in two United Kingdom centres with established ITDD services. The themes were: (1) 'making the decision to have the ITDD', family carers described very severe pain and/or sedation, in which the suffering individual would try anything; (2) 'timing and knowing they were having the best', ITDD and the associated increased access to pain and palliative care services, meant relatives felt everything possible was being done, making the situation more bearable; (3) 'was it worth it?', the perceived benefits were weighed up against the inconveniences and side effects of the ITDD; family carers judged the ITDD to be of overall benefit, despite side effects, where it had enabled the individual to be themselves through their final illness and dying phase.

Conclusion ITDD was perceived to be of greatest value when it improved QOL for patients by reducing pain and systemic side effects, thus enabling individuals to be themselves for as long as possible; under these circumstances significant side effects were judged to be acceptable. The family carers conveyed comfort in knowing that the patient was receiving what they felt was the best available management, support and specialist care. These findings should inform discussions between patients, physicians and palliative care teams around the management of severe uncontrolled pain in advanced malignancy.

\section{P-55 HIGH FIDELITY SIMULATION TRAINING IN END OF LIFE CARE; ONE CHANCE TO GET IT RIGHT}

Rina Patel, Sally Middleton, Martine Meyer, Jennifer Blair. Epsom and St Helier University Hospitals NHS Trust, Carshalton, UK

\subsection{6/bmjspcare-2017-00133.55}

Background High fidelity simulation uses technologicallyadvanced manikins to simulate complex clinical situations to train professionals in technical skills and human factors in a multidisciplinary group. We have expanded the use of simulation training (SIM) to educate about end of life care (EOLC).

Aims To evaluate the impact SIM training at Epsom and $\mathrm{St}$ Helier University Hospitals NHS Trust (ESTH) has on participant confidence, understanding and skills in EOLC and their perception of their role in end of life care.

Methods A 1 day end of life care course was initially designed by The Simulation and Interactive Learning Centre at Guy's and St Thomas' Hospital and subsequently delivered twice at ESTH. The ESTH Team adapted the scenarios and held a further 6 courses from January 2015 to June 2016. Five scenarios covered subjects including the 5 priorities for care of the dying person, individualised care planning, cultural beliefs, the Mental Capacity Act, compassion and communication.

The courses took place in the Simulation Centre, allowing a realistic ward or home setting, utilising a professional actor to enhance the scenarios.

Participants were given pre and post course questionnaires with free text sections. Information from these was used to identify themes.

Results 63 nurses and doctors of varying seniority attended the courses. Over half of the participants had worked in the NHS for at least a decade. Analysis of the data revealed that high fidelity simulation had a significant impact on the participants' confidence, understanding and skills in EOLC and their perception of their role in EOLC.

Additionally, the free text sections identified learning in patient centred care, communications skills, honesty and the importance of cultural factors.

Conclusion Use of high fidelity simulation to teach EOLC had a positive impact on the participants. We are now looking to expand the course into community settings.

\section{P-56 FAST TRACK DISCHARGE TO PREFERRED PLACE OF CARE - IDENTIFYING MODIFIABLE DELAYS}

Rina Patel, Felicity Beard, Martine Meyer. Epsom and St Helier University Hospitals NHS Trust, Carshalton, UK

\subsection{6/bmjspcare-2017-00133.56}

Background The need to facilitate dying patients spending time in their preferred place of care (PPC) was highlighted in the Department of Health's Review of Choice in End of Life Care (2015). The Fast Track process (FT) ensures patients with a rapidly deteriorating condition can be discharged to their PPC in a timely manner with the appropriate support. 